\title{
Folate contents in human milk and casein-based and soya-based formulas, and folate status in Korean infants
}

\author{
Young-Hee Han ${ }^{1}$, Miyong Yon ${ }^{1}$, Heon-Seok $\mathrm{Han}^{2}$, Kwang-Yup Kim ${ }^{3}$, Tsunenobu Tamura ${ }^{4}$ \\ and Taisun H. Hyun ${ }^{1 *}$ \\ ${ }^{1}$ Department of Food and Nutrition, Chungbuk National University, Cheongju, Korea \\ ${ }^{2}$ College of Medicine, Chungbuk National University, Cheongju, Korea \\ ${ }^{3}$ Department of Food Science and Technology, Chungbuk National University, Cheongju, Korea \\ ${ }^{4}$ Department of Nutrition Sciences, University of Alabama at Birmingham, Birmingham, AL, USA
}

(Received 5 June 2008 - Revised 8 September 2008 - Accepted 7 October 2008 - First published online 16 December 2008)

We assessed folate nutritional status from birth to 12 months in fifty-one infants who were fed human milk (HM; $n$ 20), casein-based formula $(\mathrm{CBF} ; n$ 12) or soya-based formula (SBF; $n$ 19). Folate contents in ninety-five HM samples obtained from twenty mothers for the first 6-month period and twelve CBF and nineteen SBF samples were measured by bioassay after trienzyme extraction. Folate intake was estimated by weighing infants before and after feeding in the HM group and by collecting formula intake records in the formula-fed groups. After solid foods were introduced, all foods consumed were included to estimate folate intake. Serum folate and total homocysteine (tHcy) concentrations were determined at 5 and 12 months of age, and infant growth was monitored for the first 12 months. Mean HM folate contents ranged from 201 to $365 \mathrm{nmol} / 1$ with an overall mean of $291 \mathrm{nmol} / 1$, and the contents peaked at 2 months postpartum. HM folate contents were higher than those reported in North America. Folate contents in CBF and SBF were markedly higher than those in HM and those claimed on the product labels. The overall folate intakes in formula-fed infants were significantly higher than those in HM-fed infants, and this was associated with significantly higher folate and lower tHcy in formula-fed infants than HM-fed infants at 5 months. At 12 months, serum folate was significantly higher in the SBF group than the other groups, whereas serum tHcy and overall growth were similar among all groups.

Folate: Human milk: Casein-based formula: Soya-based formula

Human milk (HM) is the ideal food for normal growth and development of infants, and is recommended to be exclusively fed to infants for the first 6 months of life ${ }^{(1-3)}$. However, substitutes are necessary when breast-feeding is impossible, and casein-based formula (CBF) has been widely used. In addition, soya-based formula (SBF) has been used as a substitute for CBF in infants with lactose intolerance, cows' milk protein allergy or diarrhoea ${ }^{(2)}$. In Korea, two types of SBF are commercially available: one contains 'isolated soya protein', as in Europe and the $\mathrm{USA}^{(4)}$; the other contains 'soya milk', which is prepared by grinding soyabeans followed by centrifugation to remove insoluble components.

Folate is a water-soluble vitamin essential for growth and development in early life ${ }^{(5)}$, since it is involved in the biosynthesis of purines and thymidylate that are building blocks of DNA and $\mathrm{RNA}^{(6)}$. However, there have been limited reports available for folate content in SBF and folate nutritional status in infants who receive SBF. Therefore, we measured the folate content of commercially available SBF along with CBF and HM. Furthermore, we assessed the folate status and growth of infants who were fed SBF and compared with those of infants who were breast-fed or received $\mathrm{CBF}$ in the first 12 months of life.

\section{Experimental methods}

Subjects and study design

The present study was carried out from 2001 to 2004 to assess folate nutritional status and growth in infants fed HM, CBF or SBF from birth to age 12 months. The study was approved by the Institutional Review Boards of Chungbuk National University Hospital (Cheongju, Korea) and the University of Alabama at Birmingham (Birmingham, AL, USA). Informed consent was obtained from each mother after the purpose and procedures of the study were explained. Healthy pregnant women (mean age 29.6 (SD 3.2) years) were recruited for participation during the third trimester by advertisements at the maternity hospitals or prenatal care programmes in Cheongju. All subjects had neither a history of serious diseases nor pregnancy complications, and had a similar socio-economic and educational background. All mothers delivered healthy fullterm infants without apparent malformations at a mean of 39.1 (SD 1.5) weeks of gestation. The female:male ratios were 8:12, 5:7 and 13:6 in the HM, CBF and SBF groups, respectively. Mean birth weights were 3483 (SD 523), 3328 (SD 390) and 3209 (SD 403) $g$ in the HM, CBF and SBF groups, respectively. Before delivery, mothers selected their

Abbreviations: CBF, casein-based formula; HM, human milk; SBF, soya-based formula; tHcy, total homocysteine

* Corresponding author: Dr T. H. Hyun, fax +82 43267 2742, email taisun@chungbuk.ac.kr 
preference of methods of infant feeding from three options including HM ( $n$ 20), CBF ( $n$ 12) and SBF ( $n$ 19). Motherinfant pairs visited a well-baby clinic at Chungbuk National University Hospital at ages 1, 2, 4, 5, 6 and 12 months, where anthropometric measurements of infants were performed by experienced nurses using an infant automatic scale (Atom, Tokyo, Japan) and a Harpenden infant stadiometer (Holtain, Crymych, Pembs, UK).

\section{Infant formulas}

Soya-based formula (Dr. Chung's Co., Cheongju, Korea) containing over $85 \%$ of 'soya milk' was offered free of charge to the SBF group. This preparation was approved as an infant formula by Korea Food and Drug Administration, and the nutritional composition of this formula was designed according to the guidance of CODEX Alimentarius ${ }^{(7)}$; folic acid (pteroylglutamic acid) was used as a source of folate in the product. Mothers in the CBF group were asked to purchase any brand of CBF. Although many mothers in the $\mathrm{CBF}$ or SBF group preferred to supplement with HM, they were requested to mixed-feed only during the first 2 weeks postpartum. They were asked to adhere to the selected feeding method exclusively in the first 5 months and allowed to feed infants with other foods thereafter. Compliance of these practices was generally acceptable with the exception that twelve of fifty-one mothers gave infants small portions of other foods (mostly vegetable based) starting at the age of 5 months.

\section{Collection of milk and blood samples}

Milk samples were collected at 1,2, 4, 5 and 6 months of lactation in the HM group, and blood samples of infants were collected at 5 and 12 months of age. Sampling of milk and blood samples was, however, not successful from all subjects at each time point. About $10 \mathrm{ml}$ milk was collected in the HM group by manual expression directly into polystyrene tubes containing ascorbic acid (10 mg; Sigma, St Louis, MO, USA) immediately before infants were fed, and the tubes were wrapped in aluminium foil to minimise light exposure. Serum samples were separated following centrifugation within 60 min of blood drawing. Sampling of milk and blood samples was not successful from all subjects at each time point. All samples were stored at $-70^{\circ} \mathrm{C}$ until analyses. A total of thirty-one formula samples (fifteen $\mathrm{CBF}$ and sixteen SBF) were bought at local stores for folate analysis. All fifteen $\mathrm{CBF}$ were in a powder form. Of sixteen SBF, fourteen were in a 'ready-to-feed' form (liquid preparation). Eleven SBF were produced with isolated soya protein and five SBF contained 'soya milk'. The formulas for folate analysis were prepared using distilled deionised water based on the manufacturers' instructions.

\section{Assessment of dietary folate intake}

Dietary folate intakes of infants were estimated at the ages of $1,2,4,5,6,9$ and 12 months. Dietary intake data were collected using a $24 \mathrm{~h}$ dietary record for $1 \mathrm{~d}$ in the HM group and for $2 \mathrm{~d}$ in the formula groups at each time point. To measure milk intake of the HM group, infants were weighed before and after each feeding using an electronic digital balance (AD-15T; CAS, Seoul, Korea) with the capability of integrated rapid serial measurements to provide a mean weight to the nearest $1.0 \mathrm{~g}$. Each mother received detailed instructions for weighing procedures including proper use of the balance and consistent clothing and diapers before and after feedings. After introducing solid foods in the HM group, 24h dietary records including solid foods were kept. At the ages of 9 and 12 months, HM intake was estimated by multiplying the nursing frequency and the average amount of milk per nursing because it was impossible to weigh due to the limit of the balance. In the CBF and SBF groups, intakes of formulas and solid foods were recorded for $2 \mathrm{~d}$ at each time point throughout the study. Folate intake from solid foods was calculated using folate values obtained with trienzyme extraction ${ }^{(8,9)}$.

\section{Folate and total homocysteine analyses}

Serum folate concentration was determined by microbiological assay using Lactobacillus rhamnosus (formerly L. casei, ATCC $7469)$ using a microplate reader interfaced with a computer ${ }^{(10)}$. The standard used for the assay was [6RS]-5-formyltetrahydrofolate (Ca salt, Sigma), because this compound appears to be more stable than folic acid that has been traditionally used. The inter-assay CV of this assay was about $8 \%$ using pooled human plasma samples for quality control ${ }^{(10)}$. Folate contents in $\mathrm{HM}$ and formulas were measured by microbiological assay after trienzyme extraction that included $\alpha$-amylase, protease and folate conjugase treatments ${ }^{(11)}$. Serum total homocysteine (tHcy) was measured by HPLC with fluorescence detection, and the inter-assay $\mathrm{CV}$ using pooled human plasma was about $9 \%^{(12)}$. The variation for food folate assay ranged between 12 and $16 \%$, where trienzyme-treatment and microbiologicalassay procedures were involved ${ }^{(13)}$.

\section{Statistical analysis}

The results are presented as mean values and standard deviations where appropriate. The differences of the means among the groups were tested by ANOVA. When the differences were significant, Tukey's post hoc test was used to compare individual means in the three groups. The Pearson correlation coefficient was used to evaluate the associations of serum folate concentrations with folate intakes, HM folate and tHcy. All analyses were performed with SAS software (version 8.2; SAS Institute Inc., Cary, NC, USA). $P$ values less than 0.05 were considered significant.

\section{Results}

The change in HM folate contents in the first 6 months of lactation is shown in Fig. 1. The mean content was highest at 2 months (365 (SD 207) nmol/l) and declined thereafter until 6 months postpartum (201 (SD 86) nmol/l); this decline was significant $(P<0 \cdot 001$; ANOVA). The mean folate contents in $\mathrm{CBF}$ were $2 \cdot 2-2 \cdot 5$ times higher than those claimed by the manufacturers, and they were $1.8-4.9$ times higher in SBF (Table 1).

There were no significant differences in the mean volumes of milk or formula intake in the three groups of infants until the age of 6 months. Because of the differences in folate contents among the $\mathrm{HM}, \mathrm{CBF}$ and $\mathrm{SBF}$ groups, calculated 


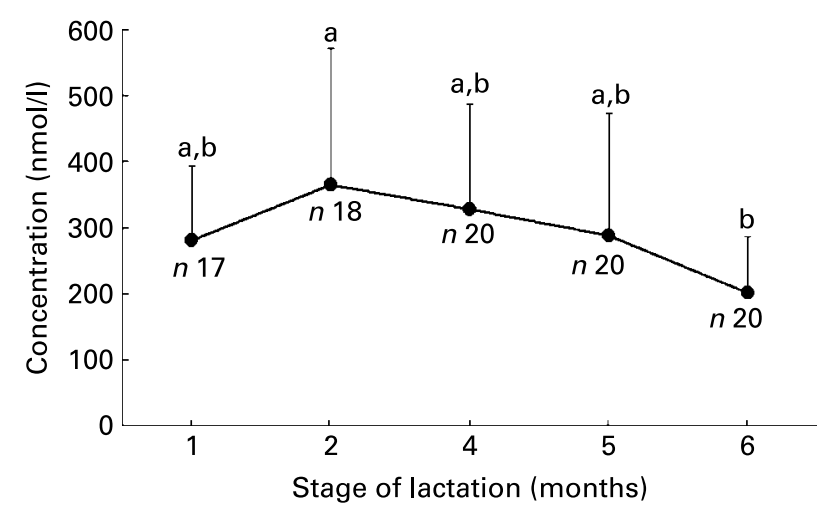

Fig. 1. Human milk folate contents in the first 6 months of lactation. Values are means, with standard deviations represented by vertical bars. The numbers of samples at each time point are shown. ${ }^{a, b}$ Mean values with unlike letters were significantly different $(P<0.05)$.

folate intakes were significantly different in these groups, and SBF-fed infants had the highest intake throughout 12 months (Fig. 2).

At the age of 5 months, serum folate concentrations in the SBF and CBF groups were significantly higher than in the $\mathrm{HM}$ group, whereas serum tHcy concentrations in the CBF group were lower than the other two groups. At the age of 12 months, serum folate concentrations of the SBF group were significantly higher than the other two groups of infants; however, serum tHcy concentrations were similar in all three groups (Table 2).

There were significant correlations between folate intakes and serum folate concentrations in all infants (three groups combined) at 5 and 12 months of life $(r 0.35 ; n 44 ; P<0.05$ and $r 0.61 ; n 30 ; P<0.001$, respectively by Pearson correlation). There were no significant correlations between serum folate and tHcy at 5 months $(r-0.33 ; n 30 ; P=0.08)$ and at the age of 12 months $(r-0.03 ; n 30 ; P=0.90)$. Furthermore, there was no significant association between serum folate concentrations and HM folate contents at the age of 5 months $(r 0.05 ; n 18 ; P=0.83)$.

The height and weight of infants were not significantly different in the three groups at 12 months. The height was between the 25 th and 75 th percentiles, and the weight was between the 10th and 90th percentiles of Korean reference values $^{(14)}$.

\section{Discussion}

Only a few research groups in the USA and Mexico have reported HM folate contents analysed by trienzyme extraction $^{(15-18)}$. Reported means of HM folate contents range from 103 to $224 \mathrm{nmol} / \mathrm{l}$ and are lower than our mean values (range $201-365 \mathrm{nmol} / \mathrm{l}$ ) in the first 6 months postpartum (Fig. 1). Although the reason for such a discrepancy is unknown, it is of interest to note that Tamura et al. ${ }^{(19)}$ reported a mean HM folate content of $320 \mathrm{nmol} / \mathrm{l}$ in Japan, which is close to the overall mean in the present study $(291 \mathrm{nmol} / \mathrm{l})$. Their mean value is the highest reported in the literature. Tamura et al. ${ }^{(19)}$ obtained the value using a microbiological assay after treatment with a crude folate conjugase preparation from hog kidney. This crude preparation might have contained sufficient amounts of $\alpha$-amylase and protease for making folates trapped in the carbohydrate and protein matrices available to the assay organism. It would be of interest to investigate whether race or dietary habits (rice is the main staple food in both Korea and Japan) plays a role in controlling folate secretion from the mammary glands.

Although folate contents in infant formulas have been reported, the assays were not done with the trienzyme extraction method ${ }^{(20-23)}$. Sweeney et al. ${ }^{(24)}$ measured the 'folic acid' content of two types of infant formulas in Ireland using the trienzyme extraction method and the L. rhamnosus microbiological assay. They found that actual 'folic acid' contents were 23.3 and $13.0 \mu \mathrm{g} / 100 \mathrm{ml}$ despite the manufacturers' claim that the amounts of folic acid added to the formulas were only 10 and $8 \mu \mathrm{g} / 100 \mathrm{ml}$, respectively. In the article, however, it is unclear whether these actual contents represented all folates including folic acid. Therefore, the present results may be the first to report on 'total' folate content in commercially available infant formulas (CBF and $\mathrm{SBF}$ ) with more suitable trienzyme extraction than traditional folate conjugase treatment alone. We found that the contents obtained with actual analysis were more than twice the content

Table 1. Folate contents of infant formulas

(Mean values and standard deviations)

\begin{tabular}{|c|c|c|c|c|c|c|}
\hline \multirow{3}{*}{ Type of formula } & \multirow{3}{*}{$\begin{array}{c}\text { Target age } \\
\text { (months) suggested }\end{array}$} & \multirow{3}{*}{$\begin{array}{c}\text { Number of } \\
\text { samples assayed }\end{array}$} & \multicolumn{4}{|c|}{ Folate content (nmol/l) } \\
\hline & & & \multicolumn{2}{|c|}{$\begin{array}{l}\text { Values on } \\
\text { products }\end{array}$} & \multicolumn{2}{|c|}{$\begin{array}{l}\text { Values } \\
\text { analysed }\end{array}$} \\
\hline & & & Mean & SD & Mean & SD \\
\hline \multirow[t]{2}{*}{ Casein-based } & $<6$ & 8 & 277 & 41 & 597 & 106 \\
\hline & $\geq 6$ & 7 & 265 & 65 & 658 & 151 \\
\hline Mean & & 15 & 271 & 52 & 625 & 128 \\
\hline \multirow[t]{3}{*}{ Soya-based ('soya milk') } & $0-5$ & 1 & 317 & - & 1272 & - \\
\hline & $6-11$ & 2 & 193 & 48 & 955 & 57 \\
\hline & $\geq 12$ & 2 & 254 & 6 & 1026 & 248 \\
\hline Mean & & 5 & 242 & 57 & 1047 & 183 \\
\hline \multirow[t]{3}{*}{ Soya-based (isolated soya protein) } & $0-5$ & 1 & 295 & - & 522 & - \\
\hline & $6-11$ & 4 & 327 & 10 & 702 & 204 \\
\hline & $\geq 12$ & 6 & 323 & 43 & 1015 & 425 \\
\hline Mean & & 11 & 322 & 32 & 856 & 372 \\
\hline
\end{tabular}




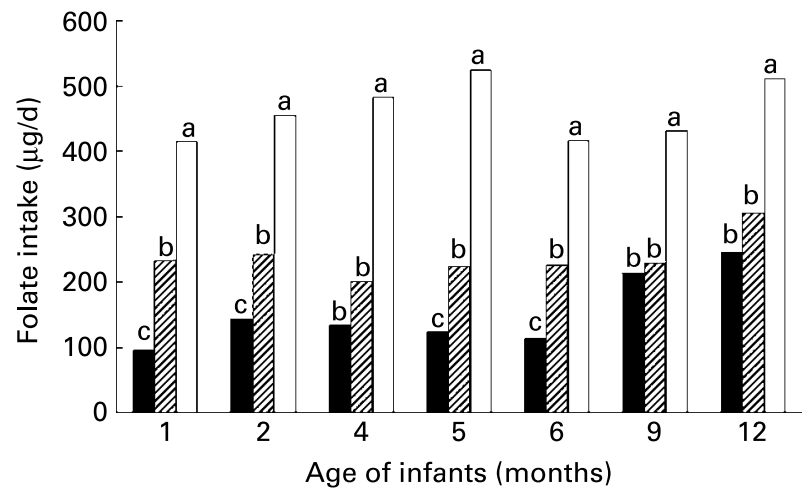

Fig. 2. Daily folate intake in the three groups of infants during the first

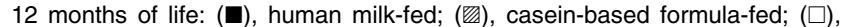
soya-based formula-fed. Values are means. ${ }^{a, b, c}$ Mean values with unlike letters were significantly different $(P<0.05)$.

claimed on the labels. It is likely that more endogenous folates in 'soya milk' were detected after the trienzyme extraction method (Table 1), and the manufacturers might have added more folic acid during the manufacturing processes. To our knowledge, there has been no report on folate contents in SBF.

Based on intakes of HM, CBF and SBF as well as of solid foods after the age of 6 months, daily total folate intakes were calculated in the three groups of infants. The infants in the HM group consistently had the lowest folate intake, and those in the SBF group had the highest folate intake (Fig. 2). Folate intakes in the $\mathrm{CBF}$ group were about twice those in the HM group during the first 6 months of life, and our finding is similar to the ratios reported by Smith et al. ${ }^{(25)}$. Furthermore, folate intakes in the SBF group were nearly twice those in the CBF group.

It has been reported that the presence of folate-binding protein in milk enhances folate bioavailability ${ }^{(25,26)}$. However, at present, it is unknown whether the bioavailability of folate in SBF is similar to those in HM or CBF. Although serum folate concentrations were significantly higher in the SBF group than the HM group at 5 months and the other two groups at 12 months (Table 2), we do not have sufficient information to judge whether these serum values reflected simply higher folate contents in SBF or the extent of bioavailability of folate in SBF. Moreover, it is unknown whether folate bioavailability is similar between SBF containing isolated soya protein and 'soya milk'. Mean folate content in SBF with 'soya milk' was slightly higher than that in SBF containing isolated soya protein. 'Soya milk' (crude preparation), however, may contain a considerable amount of folate-conjugase inhibitor that is known to be found in legumes ${ }^{(27)}$ that can reduce bioavailability of polyglutamyl folates. Nevertheless, we are concerned as to whether or not folate intakes, particularly of folic acid, an unnatural form, are excessive in infants fed SBF that contained on average three times more folate than the contents claimed by the manufacturers. We suggest that careful formulation of infant formulas should be practised.

We found that serum folate concentrations in both the CBF and SBF groups were significantly higher than those of the HM group at the age of 5 months. It has been known that

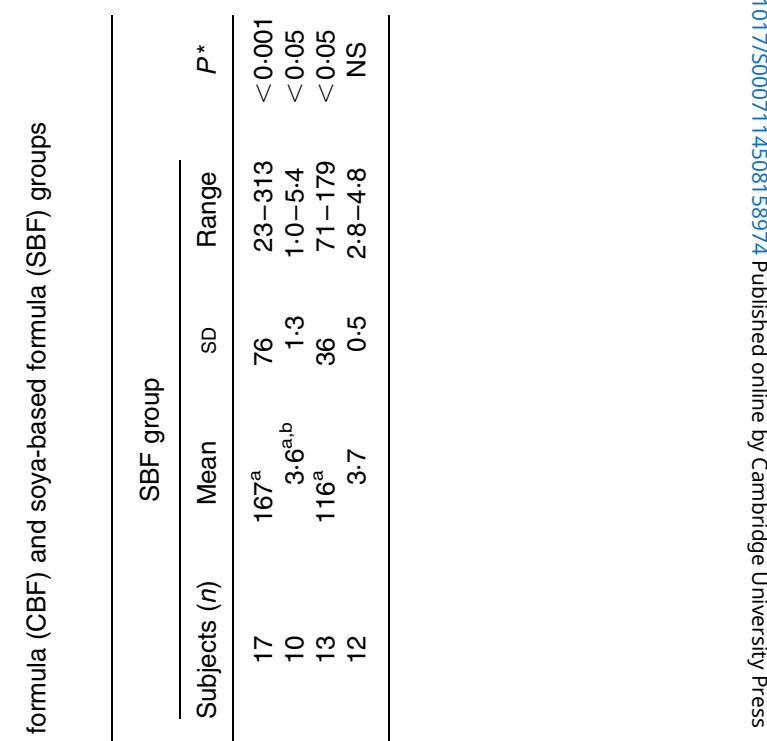


serum folate concentrations are high in the first few months of life and gradually decline thereafter ${ }^{(28)}$. In the present study, the declines were clear in both CBF and SBF groups, whereas it was unclear in the HM groups (Table 2). At present, we do not know the reason for the difference between our data of no apparent change in serum folate and those of well-established decline ${ }^{(28)}$. Plasma tHcy concentrations are generally low in early life and increase as children age ${ }^{(29,30)}$. Our values (range $1.0-6.8 \mu \mathrm{mol} / \mathrm{l}$ ) were lower than the values reported previously $^{(29)}$. The reason for these low values in the present study is unknown; however, adequate folate status as evidenced by high serum folate may be the reason. To our knowledge, there have been only a limited number of studies where differences in plasma folate and/or tHcy concentrations were investigated in infants who were fed HM or infant formulas $^{(31-33)}$, and ours may be the first study to analyse both folate and tHcy in SBF-fed infants.

The growth of the infants in the present study was within the 90th percentile of Korean reference values ${ }^{(14)}$. There were no differences in height and weight between the three groups at 12 months of age, and this observation was similar to those reported by others ${ }^{(34)}$.

In summary, we found that the overall mean HM folate content was $291 \mathrm{nmol} / \mathrm{l}$, which was higher than those reported in North America. Folate contents in CBF and SBF were markedly higher than those in HM and those claimed on the product labels; thus, the overall folate intakes in formula-fed infants were significantly higher than those in HM-fed infants, and this led to significantly higher serum folate in formula-fed infants than HM-fed infants.

\section{Acknowledgements}

We thank Kelley E. Johnston for folate and homocysteine analyses. The soya-based formula used in the present study was provided by Dr. Chung's Co. (Cheongju, Korea). Y.-H. H. was responsible for sample handling and nutrient intake assessment; M. Y. performed folate assays; H.-S. H. was responsible for subject care and blood and milk sampling; K.-Y. K. was responsible for statistical analysis; T. T. was responsible for folate and homocysteine assays and drafted the manuscript; T. H. H. was responsible for the overall investigation and drafted the manuscript.

None of the authors has anything to declare regarding conflict of interest.

\section{References}

1. Picciano MF (2001) Nutrient composition of human milk. Pediatr Clin North Am 48, 52-67.

2. O'Connor DL, Brennan J \& Merko S (2006) Formulas for preterm and term infants. In Neonatal Nutrition and Metabolism, 2nd ed., pp. 409-436 [P Thureen and W Hay, editors]. New York: Cambridge University Press.

3. World Health Organization (2002) The optimal duration of exclusive breastfeeding. Report of an expert consultation. Geneva, Switzerland. http://www.who.int/nutrition/publications/ optimal_duration_of_exc_bfeeding_report_eng.pdf (accessed June 2007).

4. Turck D (2007) Soy protein for infant feeding: what do we know? Curr Opin Clin Nutr Metab Care 10, 360-365.
5. Tamura T \& Picciano MF (2006) Folate and human reproduction. Am J Clin Nutr 83, 993-1016.

6. Wagner C (1995) Biochemical role of folate in cellular metabolism. In Folate in Health and Disease, pp. 23-42 [LB Bailey, editor]. New York: Marcel Dekker.

7. CODEX Alimentarius (2007) Standard for infant formula and formulas for special medical purposes intended for infants. Codex Stan 72 - 1981. http://www.codexalimentarius.net/ download/standards/288/CXS_072e.pdf

8. Yon M \& Hyun TH (2003) Folate content of foods commonly consumed in Korea measured after trienzyme extraction. Nutr Res 23, 735-746.

9. Han YH, Yon M \& Hyun TH (2005) Folate intake estimated with an updated database and its association to blood folate and homocysteine in Korean college students. Eur J Clin Nutr 59, 246-254.

10. Tamura T (1990) Microbiological assay of folates. In Folic Acid Metabolism in Health and Disease, pp. 121-137 [MF Picciano, ELR Stokstad and JF Gregory III, editors]. New York: Wiley-Liss.

11. Hyun TH \& Tamura T (2005) Trienzyme extraction in combination with microbiological assay in food folate analysis: an updated review. Exp Biol Med 230, 444-454.

12. Tamura T, Johnston KE \& Bergman SM (1996) Homocysteine and folate concentrations in blood from patients treated with hemodialysis. J Am Soc Nephrol 7, 2414-2418.

13. Johnston KE \& Tamura T (2004) Folate content in commercial white and whole-wheat sandwich breads. J Agric Food Chem 52, 6338-6340.

14. Korean Pediatric Society (1998) Standard growth curve 1998 (in Korean). Seoul: Korean Pediatric Society. http://www.pediatrics.or.kr/n_mem/support/view.asp?sw=1\&page=2\&no=3\&tbl_name $=$ n_data\&memchk $=\&$ search $=0 \&$ searchstring $\quad$ (accessed June 2007).

15. Lim H-S, Mackey AD, Tamura T, et al. (1998) Measurable human milk folate is increased by treatment with $\alpha$-amylase and protease in addition to folate conjugase. Food Chem $\mathbf{6 3}$, 401-407.

16. Mackey AD \& Picciano MF (1999) Maternal folate status during extended lactation and the effect of supplemental folic acid. Am J Clin Nutr 69, 285-292.

17. Villalpando S, Latulippe ME, Rosas G, et al. (2003) Milk folate but not milk iron concentrations may be inadequate for some infants in a rural farming community in San Mateo, Capulhuac, Mexico. Am J Clin Nutr 78, 782-789.

18. Khambalia A, Latulippe ME, Campos C, et al. (2006) Milk folate secretion is not impaired during iron deficiency in humans. J Nutr 136, 2617-2624.

19. Tamura T, Yoshimura Y \& Arakawa T (1980) Human milk folate and folate status in lactating mothers and their infants. Am J Clin Nutr 33, 193-197.

20. Ford JE \& Scott KJ (1968) The folic acid activity of some milk foods for babies. J Dairy Res 35, 85-90.

21. Ford JE, Porter JWG, Scott KJ, et al. (1974) Comparison of dried milk preparations for babies on sale in 7 European countries. Arch Dis Child 49, 874-877.

22. Ek J \& Magnus E (1982) Plasma and red cell folate values and folate requirements in formula-fed term infants. J Pediatr 100, $738-744$.

23. Jacoby BT \& Henry FT (1992) Liquid chromatographic determinations of folic acid in infant formula and adult medical nutritionals. J AOAC Int 75, 891-898.

24. Sweeney MR, McPartlin J, Weir DG, et al. (2005) Evidence of unmetabolised folic acid in cord blood of newborns and serum of 4-day-old infants. Br J Nutr 94, 727-730.

25. Smith AM, Picciano MF \& Deering RH (1985) Folate intake and blood concentrations of term infants. Am J Clin Nutr 41, 590-598. 
26. Picciano MF, West SG, Ruch AL, et al. (2004) Effect of cow milk on food folate bioavailability in young women. Am J Clin Nutr 80, 1565-1569.

27. Butterworth CE Jr, Newman AJ \& Krumdieck CL (1974) Tropical sprue: a consideration of possible etiologic mechanisms with emphasis on pteroylpolyglutamate metabolism. Trans Am Clin Climatol Assoc 86, 11-22.

28. Vanier TM \& Tyas JF (1966) Folic acid status in normal infants during the first year of life. Arch Dis Child 41, 658-665.

29. Monsen ALB \& Ueland PM (2003) Homocysteine and methylmalonic acid in diagnosis and risk assessment from infancy to adolescence. Am J Clin Nutr 78, 7-21.

30. Refsum H, Grindflek AW, Ueland PM, et al. (2004) Screening for serum total homocysteine in newborn children. Clin Chem 50, $1769-1784$.
31. Minet J-C, Bissé E, Aebischer C-P, et al. (2000) Assessment of vitamin B-12, folate, and vitamin B-6 status and relation to sulfur amino acid metabolism in neonates. Am J Clin Nutr 72, $751-757$.

32. Fokkema MR, Woltil HA, van Beusekom CM, et al. (2002) Plasma total homocysteine increases from day 20 to 40 in breastfed but not formula-fed low-birthweight infants. Acta Paediatr 91, 507-511.

33. Karademir F, Suleymanoglu S, Ersen A, et al. (2007) Vitamin $\mathrm{B}_{12}$, folate, homocysteine and urinary methylmalonic acid levels in infants. J Int Med Res 35, 384-388.

34. Seppo L, Korpela R, Lönnerdal B, et al. (2005) A follow-up study of nutrient intake, nutritional status, and growth in infants with cow milk allergy fed either a soy formula or extensively hydrolyzed whey formula. Am J Clin Nutr 82, 140-145. 